\title{
Benefits of CMOS Sensors for Environmental Monitoring
}

\author{
M.F. Chowdhury ${ }^{1}$, F. Udrea ${ }^{1,2}$, and J.W. Gardner ${ }^{1,3}$ \\ ${ }^{1}$ Cambridge CMOS Sensors, Deanland House, Cowley Road, Cambridge CB4 ODL, UK; \\ mohamed.chowdhury@ccmoss.com; ${ }^{2}$ University of Cambridge, Cambridge CB2 1TN, UK; \\ fu10000@cam.ac.uk ; ${ }^{3}$ University of Warwick, Coventry CV4 7AL, UK; j.w.gardner@warwick.ac.uk;
}

\begin{abstract}
The key sensor requirements for global-scale environmental monitoring are: low cost; low power; high volume production capabilities; miniature size and ubiquitous, self-powered wireless deployment. Based upon these requirements, a new generation of miniature sensors is emerging that employ nano-materials, such as metal oxides, polymers, carbon, graphene - sometimes structured as nanotubes ornanowires. Platform technologies used for depositing these gas sensing materials are commonly based on ceramic substrate, printable polymer, or silicon wafers together with a MEMS processing step. Amongst these, the most promising platform is the silicon substrate. In particular, a silicon substrate can be processed in commercial foundries with integrated CMOS circuits and is considered to be the most attractive option to enable high volume, low cost ubiquitous smart solutions with multi-sensing solutions. In this paper we give some examples of sensors that have been, or are being developed, for commercial environmental monitoring applications. Using CMOS silicon wafers for environmental sensing application offers added benefits that the sensors can be readily incorporated within portable devices such as smartphones, wearables or even in whitegoods including automotive and other purpose-built electronic systems. We also give examples of such sensors and array of sensors and highlight some of the key benefits of using CMOS sensing solutions for future environmental monitoring.
\end{abstract}

Key words: CMOS sensors, indoor air quality, wearable devices, gas sensing

\section{Introduction}

Environmental monitoring is important for the wellbeing of present and future generations. This is particularly important due to increasing pollution associated with the technological advancements being made in the developing nations. The impact of this development, if uncontrolled (as we have seen during the industrial revolutions), will have severe consequences in terms of natural ecological balance of the planet where all life on this earth depends on. The environmental monitoring process involves recording as many parameters as possible, for example, temperature, humidity, pressure, particles, toxic and greenhouse gases, liquids, air flow, nuclear radiation, electro-magnetic radiation, vibration (earth quakes), bio-medical threats, including human, animal and insects activities (e.g. movements and migration). To measure these parameters, sensors can be categorised into a number of primary groups such as: physical; chemical; mechanical; electrical and optical. With all these parameters to be measured and a diverse range of sensor categories, it can be seen that global-scale environment monitoring will be a complicated and expensive business.

To put this in to perspective, if we consider the world as a whole with a total area of $\sim 500$ million $\mathrm{km}$ square, if one sensor per $\mathrm{km}$ is sufficient, then 500 million monitors will be needed. Realistically at least 100 monitors per $\mathrm{km}$ square will be needed; this would mean some 50 billion monitors will be required. If we just need to cover a land area only which occupies roughly $29 \%$ of the Earth, in this case a minimum of 15 billion monitors will be needed. For practical global deployments of sensors for environmental monitoring, low power, energy harvesting, with wireless, will also be required. Clearly with such demanding requirements and to support high volume end application, using current discrete components solution is not a realistic option.

In order enable true ubiquitous sensing solutions for global environmental monitoring, not only the sensors need to be miniature, they need to be highly reproducible and mass produced. 


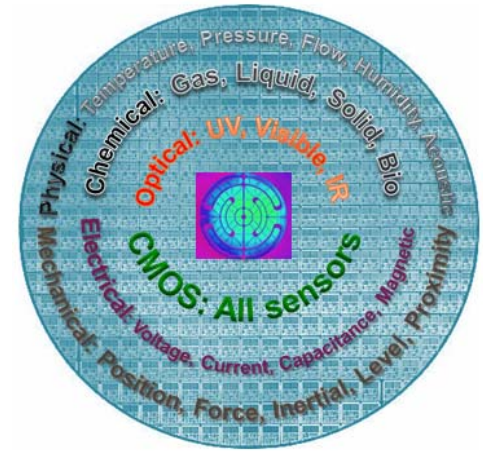

Fig 1: Types of sensors that fabricated on CMOS silicon wafer

In this paper we report how this goal can be achieved by using well-established Complementary Metal-Oxide Semiconductor (CMOS) technology, i.e. silicon as a platform to develop integrated multi-sensors to enable current and future sensing solutions. In the following sections we will describe how the CMOS process can be used as platform developing low cost sensors and highlight some of the benefits and challenges involved. Furthermore, we will present some sensing results related to Air Quality Monitoring (AQM) using CMOS sensors that are integrated in smartphone and portable handheld electronic devices.

\section{CMOS technology}

There are many types of sensors that are being mass-produced on CMOS or CMOS-compatible silicon wafers for various sensing applications. Most of these sensors have been incorporated with Micro-Electro-Mechanical Systems (MEMS) processing steps to integrate devices, such as microphones, accelerometers, pressure, temperature sensors etc. These devices are now considered as standard part of CMOS silicon wafer process flow and are being manufactured in billions of units annually [1]. For environmental monitoring, key parameters that need to be measured are: temperature; humidity; pressure; particulates and environmental gases. Amongst these parameters, most challenging ones are currently concerned with the development of low-cost miniature particulate and environmental gas sensors.

Conventional particle sensors are bulky and mainly use optical spectroscopic method of monitoring [2]. However, using MEMS micromirrors and acoustic solidly mounted resonators (SMRs) on CMOS wafers are emerging to develop low-cost chip-level particle sensors solutions [3]. Fig 2, shows of examples suspended microchannel resonator (SMR) particle sensor and SMD packaged gas sensor.
As for environmental gas sensors, there are typically at least six different technologies: Electrochemical; Calorimetric; Capacitive; Acoustic; Optical; and Optical.
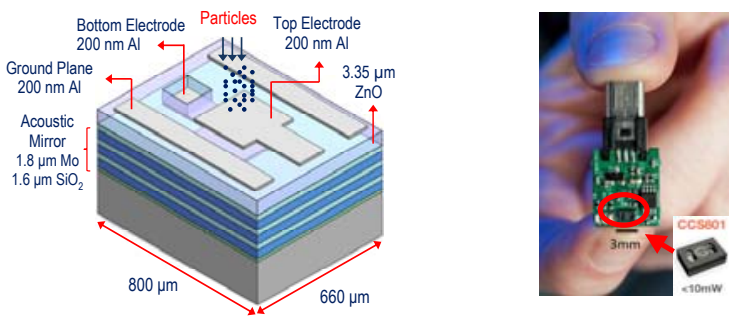

Fig 2: (a) SMR particle sensor; (b) CMOS MEMS Gas sensor demo module

Traditionally, being a solid-state device, gas sensors have been bulky and relatively expensive. Amongst them, most popular sensors are based on electrochemical technology, though compared to other technologies, they are relatively accurate and reasonably selective, but do suffer from poisoning, long term stability and lifetime issues. Table 1, shows some of the key parameters and comparative summary between various gas sensing methods.

\begin{tabular}{lllll}
\hline Parameter & EC & MOX & PA & IR \\
\hline Selectivity & Good & Poor & Good & Good \\
\hline Accuracy & Good & Good & Poor & Good \\
\hline Temperature & Good & Poor & Best & Poor \\
\hline Humidity & Poor & Poor & Poor & Poor \\
\hline Lifetime & Poor & Good & Good & Best \\
\hline Calibration & Poor & Good & Poor & Good \\
\hline Cost & High & Low & High & High \\
\hline Size & Bulky & Tiny & Big & Big \\
\hline Power & Lower & Low & High & Med \\
\hline Ubiquitous & No & Yes & No & No
\end{tabular}

EC-Electrochemical; MOX - Metal oxide; PA- Photoacoustic; IR - Infrared

Table 1: Comparison of gas sensing methods

Table 1, shows that there need to be a trade-off between performance, cost and miniaturisation. For low cost, high volume miniature gas sensing applications it can be concluded MOX sensor is the only realistic option. Yet, slow progress has been made over the last thirty years in developing alternative gas sensing solutions using metal-oxide and nano-material deposited on alumina and silicon substrates [4].

\section{CMOS Sensor manufacturing process}

To manufacture integrated metal oxide (MOX) based gas sensors; one of the key challenges 
has been that for most gas sensing applications, the sensing materials need to be heated up to at least $300^{\circ} \mathrm{C}$ and in some cases more than $600^{\circ} \mathrm{C}$. This challenge has been overcome by integrating MEMS micro-hotplate on standard CMOS wafer as show in Fig 3.

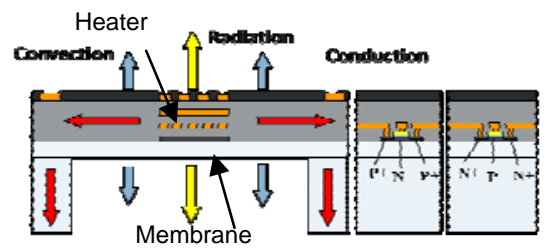

Fig 3: CMOS, MEMS micro-hotplate platform for integrated gas sensor

The micro-hotplate shown in Fig 3 is integrated with high temperature metallisation steps, such as platinum or tungsten, where heater is fabricated on a thin membrane, produced with a MEMS etching process. By placing the heater on the membrane low power, fast thermal transient response is achieved thus offering added benefit of developing sensors based on thermal modulation techniques [5]. In addition, such micro-hotplates can make good wideband infrared sources with fast switching speed $(>100 \mathrm{~Hz} @ 50 \%$ modulation depth are being used for NDIR gas sensing and spectroscopy applications.

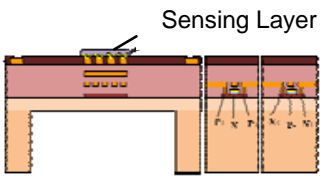

(a)

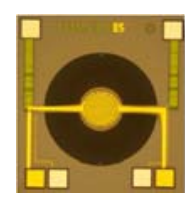

(b)

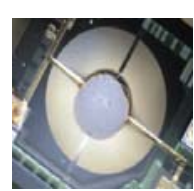

(c)
Fig 4: (a) CMOS MEMS cross-section with sensing layer; (b) Die with post-CMOS gold electrodes; (c) MOX deposited die

Fig 4 shows critical CMOS gas sensor process steps. Here a MOX sensing layer is deposited on the membrane with gold electrodes, where the resistance of the material is measured to determine the gas concentration. The working principals of MOX sensors are well described in the literature, and best described by a redox reaction between the target gases and chemisorbed oxygen ions $\mathrm{O}^{-}, \mathrm{O}_{2}^{-}, \mathrm{O}^{2-}$. [6]. The oxygen creates depletion regions that limit electron mobility across the granular nanostructure. This results in either an increase (oxidising gases) or decrease (reducing gases) of the film resistance (hence the term chemoresistive) when the target gas is present. The response is a function of the operating temperature that can also affect sensitivity, selectivity, stability, and response time [7].

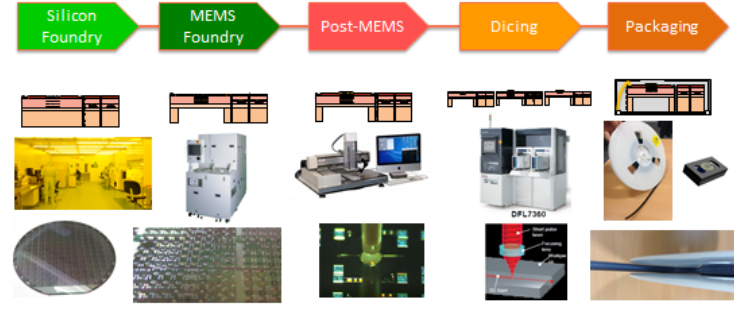

Fig 5: CMOS MOX sensor production process flow

Volume production flow for CMOS, MEMS sensors, particularly where post-CMOS processing is involved is shown in Fig 5. Here, for gas sensing application, key challenges are reliable sensing layer deposition on the membrane. As this process is not an integral part of CMOS process flow, as a result this will lead to certain yield loss as multiple supplychain is involved as shown in Fig 6.

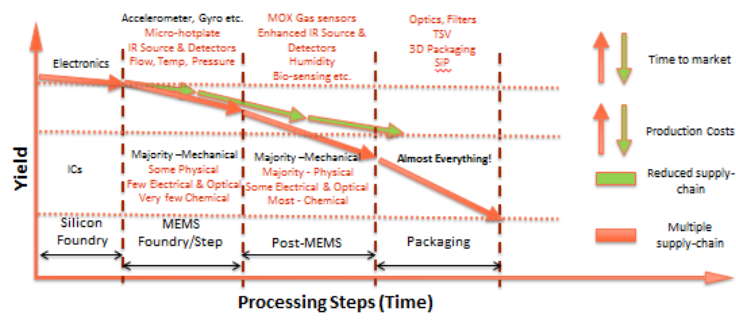

Fig 6: Impact on yield as multiple supply chain used

In order to reduce this yield loss and to allow high volume multi-sensing production capabilities, the challenge is to develop a unified process flow of all sensors development stages within the same silicon foundry. Some of the key steps are shown in Fig 7.

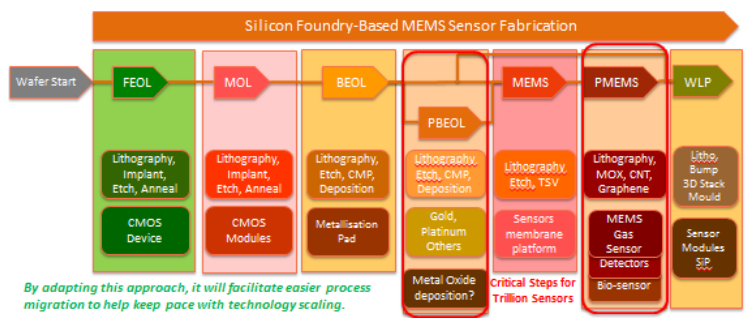

Fig 7: CMOS, MEMS sensor process flow

This process will typically involve front-end-ofline (FEOL), Mid-end-of-line (MEOL), back-endof-line (BEOL), post-back-end-of-line (PBELO), MEMS, Post-MEMS and wafer-level-processing (WLP). As demand for sensors increases, a unified process flow will evolve leading to yield improvements and enhanced sensor performance [8].

\section{CMOS Sensors for environment monitoring}

Recently a number of low cost environmental sensors are emerging in smartphones, portable and wearable devices. One such example of a sensor incorporated within a smartphone that 
has been developed by Cambridge CMOS Sensors Ltd (UK) is shown in Fig 8.

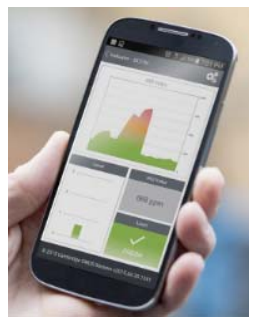

(a)

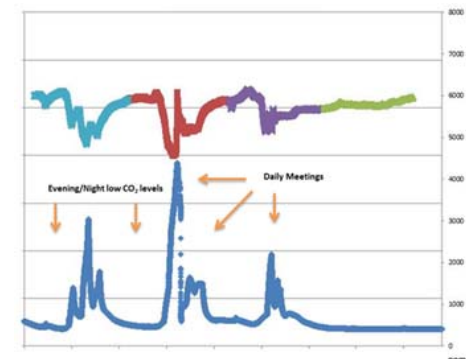

(b)
Fig 8: (a) Integrated CMOS environmental sensor in a smartphone; (b) Air quality response in office to VOC and $\mathrm{CO}_{2}$

In this example, a CMOS MEMS MOX sensor is packaged on $2 \mathrm{~mm} \times 3 \mathrm{~mm}$ SMD footprint, and assembled on a PCB directly inside the smartphone where the environment monitoring performed by running an application software (Fig 8(a)). In Fig 8(b), the same sensor is placed in a data logging module and results of VOC and equivalent $\mathrm{CO}_{2}$ level recorded in a meeting room is shown. Data were recorded over a 4-day period (colour coded: blue, red, purple and green) as shown in Fig 8(b).

For harsh environmental monitoring, comparison of EC sensor with MOX sensor for monitoring $\mathrm{SO}_{2}, \mathrm{H}_{2} \mathrm{~S}, \mathrm{CO}$ and $\mathrm{NH}_{3}$ was carried out in a poultry farm. The sensor module was dual/battery operated and provide with zigbee wireless interface where data was remotely logged using a laptop computer (see Fig 9).

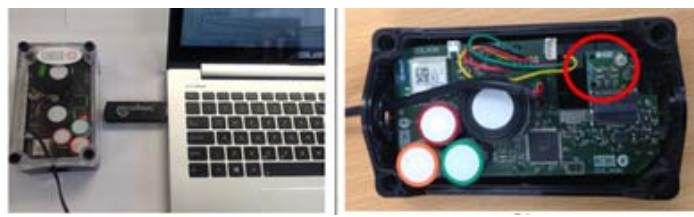

Fig 9: Combined EC and MOX sensor module

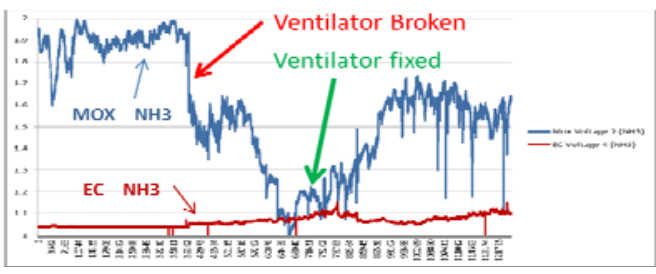

Fig 10: Comparison of $\mathrm{NH}_{3}$ response EC vs $\mathrm{MOX}$

The sensor module was deployed on a farm over a 3 month period where, in addition to above gases, $\mathrm{CO}_{2}$, atmospheric pressure, humidity and temperature were also recorded. Results in Fig 10, shows raw ADC output of the sensor $\mathrm{NH}_{3}$ sensor response only $\left(\mathrm{CO}, \mathrm{H}_{2} \mathrm{~S}\right.$ and $\mathrm{SO}_{2}$ sensor showed "zero" as expected). The graph shows that the MOX sensor responded well to $\mathrm{NH}_{3}$, but as the ventilator was broken (as shown by red arrow), the concentration level increased very rapidly compared to EC sensor. When the ventilator was fixed $\mathrm{NH}_{3}$ concentration level fell as shown by EC sensor.

\section{Conclusion}

Key benefits of CMOS sensing solutions summarised as follows:

1. The silicon process perfected over more than 60 years.

2. Proven to go beyond more than Moore's law.

3. Billions of dollars invested in technology, methodologies and instrumentations.

4. Billions of sensors already in production (microphone, accelerometer, pressure.).

5. Technology offers true high volume production capabilities.

6. Offers scalability for lasting product life cycle and for future innovations.

7. Integrated solution to enable smart multisensing capabilities.

8. Wafer-level processing for 3D, heterogeneous packaged solutions.

9. Provides high yield, low cost, ultraminiature, low power solution.

10. Multi-sensing to enable system-on-a-chip solution!

\section{Acknowledgements}

Authors would like to acknowledge the support of Cambridge CMOS Sensors team and COST Action TD1105 EuNetAir.

\section{References}

[1] E. MOUNIER, Future of MEMS: a Market \& Technologies Perspective, Yole Development, MEMS Tech Seminar 2014

[2] HYDAC Filtertechnik $\mathrm{GmbH}, \quad$ Particle Measurement Technology in Practice. From Theory to Application. www.hydac.com

[3] A. Hajjam et al, "Individual Air-Borne Particle Mass Measurement Using High-Frequency Micromechanical Resonators", IEEE SENSORS JOURNAL, VOL. 11, NO. 11, NOVEMBER 2011

[4] G. Neri, First Fifty Years of Chemoresistive Gas Sensors, Chemosensors 2015, (3, 1-20; doi:10.3390/chemosensors3010001)

[5] F. Udrea, J.W. Gardner, et al. "Design and simulations of SOI CMOS micro-hotplate gas sensors", Sensors and Actuators B, 78 (2001), pp. 180-190.

[6] X. Liu et al., A Survey on Gas Sensing Technology, Sensors 2012, (12, 9635-9665; doi:10.3390/s120709635)

[7] R. Gutierrez-Osuna, Adaptive Microsensor Systems, Annual Review of Analytical Chemistry 2010.3:255-276

[8] L. Xu et al., Micro/Nano Gas Sensors: A New Strategy Towards In-Situ Wafer-Level Fabrication of High-Performance Gas Sensing Chips www.nature.com/scientificreports, May 2015 